\title{
Septischer Schock und systemisches Entzündungsreaktions-Syndrom - Diagnostik
}

\section{U. Müller-Werdan}

\section{Septic shock and systemic inflammatory response syndrome - diagnostics}

\section{Alktuelle Sepsis- und SIRS-Definitionen}

Das facettenreiche klinische Bild der Sepsis kann die Diagnosestellung schwieriger als erwartet machen. Noch mehr ist dies der Fall, wenn man den Schweregrad abschätzen und den Erfolg der durchgeführten Therapie beurteilen will. Dies trifft in noch stärkerem Maße für das systemische Entzündungsreaktions-Syndrom (SIRS) nicht-infektiöser Genese zu.

Aus diesem Grunde war es zu begrüßen, dass die intensivmedizinischen Gesellschaften die in Tab. 1 aufgeführten Sepsis- und SIRSDefinitionen präzisiert haben (9). Für den Praktiker sind diese Definitionen qualitativer Art aufgrund der geringen Spezifität allerdings nur eine bedingte Hilfe (18), und zur Schweregradeinschätzung und Verlaufsbeurteilung sind sie überhaupt nicht geeignet. Im Verständnis der deutschen Medizin kommt diese Sepsisdefinition darüber hinaus einer Inflation des Sepsisbegriffes gleich. Für unser Verständnis ist Sepsis nach wie vor eine schwere, lebensbedrohliche Erkrankung. Diesen Charakter verliert die Sepsis jedoch, wenn man der Definition der Konsensus-Konferenz folgt. Viel eher würden wir die Diagnose einer Sepsis stellen können, wenn die Situation der „severe sepsis“ in der Definition der Konsensus-Konferenz vorliegt. Der schwere Verlauf wird durch Manifestation von Hypotension/Minderperfusion und/oder Dysfunktion vitaler Organsysteme definiert. Hypotension/Hypoperfusion kann bis zum Bild des manifesten septischen Schocks fortschreiten, aus der initialen Dysfunktion vitaler Organsysteme kann sich ein manifestes Organversagen entwickeln. So können infektiöses und nicht-infektiöses SIRS in Schock oder Multiorgan-Dysfunktionssyndrom münden (siehe unten).

\section{Sepsis-Stadien nach Bone (3)}

Nachdem die proinflammatorische Phase des septischen Geschehens (SIRS) in den letzten Jahren ganz im Vordergrund des Interesses der Sepsisforschung stand, wird in letzter Zeit dem antiinflammatorischen CARS und dem mehrere pro- und antiinflammatorische Episoden umfassenden MARS mehr Beachtung geschenkt (Tab. 1). Durch eine antiinflammatorische Gegenreaktion können das Krankheitsgeschehen und die Prognose des
Patienten durch eine monozytäre Immunparalyse geprägt sein. Aus der diagnostischen Trennung von SIRS und MARS könnten sich zukünftig differentialtherapeutische Ansätze ergeben.

\section{Quantifizierung des Schweregrades der Sepsis}

\section{Scoresysteme}

Obwohl derzeit routinemäßig noch wenig eingesetzt, können Scoresysteme bei Diagnosestellung, Schweregradklassifizierung und Verlaufsbeurteilung von Sepsis und septisch bedingtem Multiorgan-Dysfunktionssyndrom hilfreich sein (14). Die Scorehöhe korreliert dabei mit dem Schweregrad der Sepsis bzw. dem Sepsisbedingten Multiorgan-Dysfunktionssyndrom und damit mit der Prognose der Patienten: je höher der Scorewert, umso höher die Letalität. Jedoch kann kein Score 100\% prognostische Treffsicherheit im Individualfall erreichen. Die Hauptlimitation des auf die Prognoseabschätzung abzielenden Einsatzes von Scores ist die Tatsache, dass eine Aussage zum Letalitätsrisiko nur für Patientengruppen und nicht für den individuellen Patienten anwendbar ist (14). Die von einem Score erbrachte Risikoeinschätzung entspricht einer statistischen Wahrscheinlichkeit mit der Sensitivität und Spezifität der ROC-Kurve der Gruppe und keiner individuellen Prognose. Scores werten verschiedene leicht fassbare Parameter. Je „pathologischer" die Ausprägung eines Befundes ist, desto mehr Punkte werden unter der Hypothese vergeben, dass eine stärkere Abweichung von der Norm mit einem höheren Schweregrad und somit schlechteren Prognose einhergeht (14): Von den zahlreichen publizierten Scores (Übersicht in (22)) bieten sich aus Praktikabilitätsgründen vor allem der Sepsis-Score nach Elebute und Stoner (Tab. 2) sowie die „Schweregrad der Erkrankung“-Scores APACHE II (Tab. 3) und SAPS II an. Diese Scores bedienen sich einfacher Parameter, die täglich auf der Intensivstation erhoben werden.

Zusätzlich zu den bereits bestehenden Organversagen-Scores wurde von der Europäischen Intensivmedizinischen Gesellschaft der SOFAScore („sepsis-related organ failure assessment score“) entwickelt (17). Er erfasst die wichtigsten Organdysfunktionen mit jeweils einem einzelnen Parameter und teilt den Schweregrad der Organdysfunktion entsprechend der Abweichung dieses Parameters von der 
Tab. 1 Terminologie und Definitionen.

Infektion: Entzündliche Gewebereaktion auf Mikroorganismen oder Invasion von Mikroorganismen in normalerweise steriles Gewebe.

Bakteriämie: Vorhandensein vitaler Bakterien im Blut; die Anwesenheit von Viren, Pilzen, Parasiten oder anderen Pathogenen in der Blutbahn sollte entsprechend benannt werden.

SIRS („,systemic inflammatory response syndrome“, „systemisches Entzündungsreaktions-Syndrom "): Systemisch-entzündliche Reaktion auf verschiedene schwere klinische Insulte, charakterisiert durch zwei oder mehr der folgenden Symptome:

Körpertemperatur $>38,0^{\circ} \mathrm{C}$ oder $<36,0^{\circ} \mathrm{C}$,

. Herzfrequenz $>90 / \mathrm{min}$

3. Atemfrequenz $>20 /$ min oder $\mathrm{p}_{\mathrm{a}} \mathrm{CO}_{2}<32 \mathrm{mmHg}$

4. Leukozyten $>12000 / \mathrm{mm}^{3}$ oder $<4000 / \mathrm{mm}^{3}$, oder $>10 \%$ unreife (stabförmige) Formen

CARS („compensatory anti-inflammatory response syndrome“): Kompensatorisches anti-inflammatorisches Reaktions-Syndrom, das sich - im Anschluss an die proinflammatorische Phase - als Anergie, als erhöhte Empfindlichkeit gegenüber Infektionen oder als beides manifestiert.

MARS („mixed antagonistic response syndrome“): Antagonistisches Reaktionssyndrom, das sich aus mehreren SIRS- und CARS-Phasen zusammensetzt.

Sepsis: Systemische Reaktion aufeine Infektion, charakterisiert durch zwei oder mehr der folgenden, durch die Infektion hervorgerufenen Symptome:

1. Körpertemperatur $>38,0^{\circ} \mathrm{C}$ oder $<36,0^{\circ} \mathrm{C}$,

2. Herzfrequenz $>90 / \mathrm{min}$

. Atemfrequenz $>20 /$ min oder $\mathrm{p}_{\mathrm{a}} \mathrm{CO}_{2}<32 \mathrm{mmHg}$

4. Leukozyten $>12000 / \mathrm{mm}^{3}$ oder $<4000 / \mathrm{mm}^{3}$, oder $>10 \%$ unreife (stabförmige) Formen

Schwere Sepsis: Sepsis, assoziiert mit Organdysfunktion, Minderperfusion oder Hypotonie. Minderdurchblutung und Durchblutungsstörungen können beinhalten, sind aber nicht beschränkt auf: Laktatazidose, Azidose, Oligurie oder eine akute Änderung der Bewusstseinslage.

MODS („multiple organ dysfunction syndrome“): Dermaßen geänderte Organfunktion bei Akutkranken, dass die Homöostase ohne Intervention nicht mehr aufrechterhalten werden kann.

Sepsis-induzierte Hypotonie: Systolischer Blutdruck $<90 \mathrm{mmHg}$ oder Reduktion um $\geq 40 \mathrm{mmHg}$ des Ausgangswerts bei Fehlen anderer Hypotonieursachen.

Septischer Schock: Sepsisinduzierter Schock mit Hypotonie trotz adäquater Volumensubstitution, einhergehend mit Hypoperfusionszeichen oder Organdysfunktionszeichen; letztere können beinhalten, sind aber nicht beschränkt auf: Laktatazidose, Azidose, Oligurie oder eine akute Änderung der Bewusstseinslage. Patienten, die infolge einer Therapie mit inotropen oder vasokonstriktiven Substanzen nicht mehr hypotensiv sind, aber dennoch Zeichen der Hypotension oder Organdysfunktion aufweisen, werden trotzdem dem Stadium septischer Schock zugeordnet.

Refraktärer septischer Schock: Septischer Schock ohne rasches Ansprechen auf Volumengabe (z.B. $500 \mathrm{ml} \mathrm{NaCl}$ in $30 \mathrm{~min}$ ) und Vasopressoren (z.B. Dopa$\min >10 \mu \mathrm{g} / \mathrm{kg} \mathrm{KG} / \mathrm{min}$ ).

Akute septische Kardiomyopathie: Myokardschädigung im Rahmen einer Sepsis mit der Folge einer im Verhältnis zum systemischen Gefäßwiderstand verminderten Pumpfunktion des Herzens.

Zusammenstellung nach (9, 2,19)

Norm ein. Im Falle der Herz-Kreislauf-Dysfunktion wird die Bewertung anhand der zur Blutdruckstabilisierung notwendigen Katecholamintherapie vorgenommen. Dieser einfach zu handhabende Score soll zur standardisierten Schweregradbeschreibung des sepsisbedingten MODS in quantifizierbarer Form beitragen.

\section{Systemischer Gefäßwiderstand}

Der stark erniedrigte systemische Gefäßwiderstand ist ein sehr wesentliches Kriterium hoher Spezifität und Sensitivität der Sepsis-verursachten Gefäßschädigung. Werte von 500200 dyn $\times$ sek $\times \mathrm{cm}^{-5}$ sind - von wenigen Ausnahmen abgesehen (z.B. Leberzirrhose, Therapie mit vasodilatierenden Pharmaka, z.B. Phosphodiesterasehemmer, anaphylaktischer Schock) - ein untrügliches Zeichen für das Vorliegen von Sepsis und septi-
Tab. 2 Prinzip der Berechnung des Sepsisscores nach Elebute und Stoner (14)

\begin{tabular}{|c|c|c|c|c|c|c|c|}
\hline \multicolumn{2}{|c|}{ Anomal niedrig } & \multirow{2}{*}{$\begin{array}{l}\text { Normbereich bzw. } \\
\text { unauffällig } \\
\text { Punkte: }\end{array}$} & \multicolumn{5}{|c|}{$\begin{array}{l}\text { Anomal hoch bzw. } \\
\text { pathologischer Befund }\end{array}$} \\
\hline \multicolumn{2}{|l|}{ Punkte: } & & \multicolumn{5}{|c|}{ Punkte } \\
\hline \multirow[t]{8}{*}{3} & 1 & 0 & 2 & 3 & 4 & 5 & 6 \\
\hline & & Infektzeichen & & & & & \\
\hline & & Wundinfekt & & & \pm & & \\
\hline & & Peritonitis & & & & & \pm \\
\hline & & Pneumonie & & & & & \pm \\
\hline & & Tiefer Abszess & & & & & \pm \\
\hline & & Harnwegsinfekt & & & \pm & & \\
\hline & & Pyrexie & & & & & \\
\hline \multirow[t]{10}{*}{$<36,0$} & & Temperatur $\left({ }^{\circ} \mathrm{C}\right)$ & & $>39,0$ & & & \\
\hline & \pm & Zusatzpunkte* & & & & & \\
\hline & & $\begin{array}{l}\text { Organversagen und } \\
\text { Laborwerte }\end{array}$ & & & & & \\
\hline & & Beatmung & \pm & & & & \\
\hline & & metabolische Azidose & \pm & & & & \\
\hline & & Neurologie & & \pm & & & \\
\hline & & DIC & & \pm & & & \\
\hline & & Kreatinin & & $>3,5$ & & & \\
\hline & & Bilirubin & $>3,5$ & & & & \\
\hline & & Blutkultur & & & \pm & & \\
\hline \multirow[t]{3}{*}{$<2,5$} & & Leukozyten & $>30$ & & & & \\
\hline & $<100$ & Thrombozyten & & & & & \\
\hline & $<7$ & Hämoglobin & & & & & \\
\hline$<25$ & & Albumin & & & & & \\
\hline
\end{tabular}

schem Schock. Eine dermaßen ausgeprägte Vasodilatation ist auch durch Katecholamingabe kaum mehr im Sinne einer Erhöhung des systemischen Gefäßwiderstandes zu beeinflussen.

\section{Serum-Procalcitonin als quantitativer Parameter für die Diagnose „Infektion“}

Von den zahlreichen getesteten Laborparametern scheint das Serum-Procalcitonin (PCT) derzeit als Sepsismarker am Erfolg versprechendsten (15). Bei Patienten mit PCT-Spiegeln $\leq 0,5 \mathrm{ng} / \mathrm{ml}$ ist die Diagnose einer Sepsis unwahrscheinlich; auch wenn es keinen definierten „Cut-off"-Punkt gibt, so sind bei Intensivpatienten Werte über 1,0-1,5 ng/ml verdächtig für eine Sepsis; jedoch können die PCT-Werte auch nach schweren Traumata oder größeren Operationen ansteigen (15).

In einer Studie mit 337 SIRS-Patienten (1) wiesen Patienten mit mikrobiell gesicherter Sepsis Procalcitonin-Spitzenwerte von $30 \mathrm{ng} / \mathrm{ml}$ auf, während sich bei den SIRS-Patienten ohne Sepsis Procalcitonin-Werte von $0,1 \mathrm{ng} / \mathrm{ml}$ oder darunter fanden.

Aufbauend auf diesen Ergebnissen sind folgende Schlussfolgerungen gezogen worden:

Procalcitonin-Werte von $0,1-0,5 \mathrm{ng} / \mathrm{ml}$ : schwere mikrobielle Infektion ist unwahrscheinlich (Sensitivität 91\%, Spezifität 25\%; positiv prädiktiver Wert 39\%, negativer prädiktiver Wert $86 \%$ ); 
Tab. 3 Prinzip der Berechnung des APACHE-II-Scores (19).

\begin{tabular}{|c|c|c|}
\hline $\begin{array}{l}\text { Anomal niedrig } \\
\text { bis } 4 \text { Punkte* }\end{array}$ & $\begin{array}{l}\text { Normbereich } \\
\text { OPunkte }\end{array}$ & $\begin{array}{l}\text { Anomal hoch } \\
\text { bis } 4 \text { Punkte* }\end{array}$ \\
\hline$<29,9^{\circ} \mathrm{C}$ & Körpertemperatur & $\geq 41,0^{\circ} \mathrm{C}$ \\
\hline$\leq 49 \mathrm{mmHg}$ & Mittlerer Blutdruck & $\geq 160 \mathrm{mmHg}$ \\
\hline$\leq 39 / \min$ & Herzfrequenz & $\geq 180 / \mathrm{min}$ \\
\hline$\leq 5 / \min$ & Atemfrequenz & $\geq 50 / \mathrm{min}$ \\
\hline$<55 \mathrm{mmHg}$ & $\mathrm{PaO}_{2}$ & \\
\hline$\geq 500 \mathrm{~mm} \mathrm{Hg}$ & $\mathrm{A}-\mathrm{aDO}_{2}$ & \\
\hline$<7,15$ & PH arteriell & 7,70 \\
\hline$\triangle 10 \mathrm{mmol} / \mathrm{l}$ & Serum-Natrium & $\geq 180 \mathrm{mmol} / \mathrm{l}$ \\
\hline$<2,5 \mathrm{mmol} / \mathrm{l}$ & Serum-Kalium & $\geq 7,0 \mathrm{mmol} / \mathrm{l}$ \\
\hline$<0,6 \mathrm{mg} / 100 \mathrm{ml}$ & Serum-Kreatinin & $3,5 \mathrm{mg} / 100 \mathrm{ml}$ \\
\hline \multicolumn{3}{|l|}{2 Punkte } \\
\hline$<20 \%$ & Hämatokrit & $\geq 60 \%$ \\
\hline$<1,0 G / l$ & Leukozyten & $\geq 40 \mathrm{G} / \mathrm{I}$ \\
\hline & \multicolumn{2}{|l|}{ Glasgow Coma Scale } \\
\hline \multicolumn{3}{|c|}{$\begin{array}{l}\text { A-aDO } \text { : alveolo-arterielle Sauerstoffdruckdifferenz } \\
\text { Hypothese:Je höher der Punktwert, desto schwerer die Erkrankung. } \\
{ }^{*} \text { Punktvergabe bis } 4 \text { Punkte je nach Abweichung von der Norm }\end{array}$} \\
\hline
\end{tabular}

Procalcitonin-Werte $>0,5 \mathrm{ng} / \mathrm{ml}$ : Infektion sehr wahrscheinlich (Sensitivität $60 \%$, Spezifität $79 \%$; positiv prädiktiver Wert $61 \%$, negativ prädiktiver Wert 78\%).

De Werra et al. (4) unterzogen verschiedene Laborparameter Procalcitonin, TNF- $\alpha$, lösliche TNF-Rezeptoren, IL-6, Nitrit/Nitrat, lösliche TNF-Rezeptoren - einem Vergleich bei Patienten mit septischem Schock, kardiogenem Schock oder Pneumonie. Als Ergebnis ihrer Studie hielten die Autoren fest, dass erhöhte Werte für Nitrit/Nitrat und Procalcitonin am besten geeignet waren, Patienten mit septischem Schock zu definieren.

Harbarth et al. fanden bei einem „Cut-off“ für Procalcitonin von 1,1 $\mathrm{ng} / \mathrm{ml}$ eine Sensitivität von $97 \%$ bei einer Spezifität von 78\% zur Diskriminierung zwischen SIRS und Sepsis bei Intensivpatienten (8). Der Median des Procalcitonin-Werts bei Aufnahme betrug in dieser Studie 0,6 $\mathrm{ng} / \mathrm{ml}$ bei SIRS, 3,5 $\mathrm{ng} / \mathrm{ml}$ bei Sepsis, $6,2 \mathrm{ng} / \mathrm{ml}$ bei schwerer Sepsis und $21,3 \mathrm{ng} / \mathrm{ml}$ beim septischen Schock. Eine weitere Studie fand jedoch einen mittleren Procalcitonin-Wert von $5,45 \mathrm{ng} / \mathrm{ml}$ bei SIRS-Patienten am Aufnahmetag (6), so dass eine Diskriminierung zur Sepsis mit Procalcitonin allein bei diesem Patientenkollektiv nicht möglich war.

\section{Zytokin-Serumspiegel als prognostische Parameter (5)}

Im Serum septischer Patienten können IL-1 und IL-1-Rezeptor-Antagonist, IL-6, TNF- $\alpha$ und die löslichen TNF-Rezeptoren, IL-8 und IL10 verlässlich bestimmt werden. Im Vergleich mit anderen Zytokinen ergibt sich für die IL-6-Serumspiegel die höchste prognostische Aussagekraft zur Beurteilung der Sepsis sowie eine Korrelation mit dem Schweregrad der Organdysfunktion. Ein IL-6-Spiegel von größer als $1000 \mathrm{ng} / \mathrm{ml}$ wurde als Einschlusskriterium für die MONARCS-Studie zur Behandlung von Sepsispatienten mit einem monoklonalen anti-TNF- $\alpha$-Antikörper verwendet (11), um eine Stratifizierung der Sepsispatienten vorzunehmen.

Die klinische Wertigkeit von TNF $\alpha$-Serumspiegel septischer Patienten ist eingeschränkt aufgrund der starken Streubreite der gemesse- nen Werte, die vermutlich durch die relativ kurze biologische Halbwertszeit des TNF- $\alpha$ zustande kommt. Dagegen sind die Blutspiegel der löslichen TNF-Rezeptoren (p55 und p75) aufgrund ihrer längeren biologischen Halbwertszeit besser geeignet zur Beurteilung des Letalitätsrisikos, wie bei Patienten nach herzchirurgischen Operationen gezeigt werden konnte $(12,13)$. Zytokinspiegelmessungen gehören derzeit noch nicht zur klinischen Routine.

\section{Bestimmung der Immunkompetenz}

Aktuelle Bestrebungen gehen dahin, die Immunkompetenz des Patienten in der Sepsis diagnostisch zu erfassen, um proinflammatorische Phasen gegen antiinflammatorische Gegenreaktionen in der Sepsis abzugrenzen. Sowohl die Bestimmung der monozytären HLA-DR-Expression als auch die Ex-vivo-TNF $\alpha$-Produktion nach LPS-Stimulation sind hierfür geeignete Parameter (16).

kurzgefasst: Zur Abschätzung des Schweregrades einer Sepsis können Score-Systeme und systemischer Gefäßwiderstand herangezogen werden. Scoresysteme bedienen sich einfacher Kriterien. Sie können keine individuelle Prognoseabschätzung liefern.

\section{Multiorgan-Dysfunlktionssyndrom und Mulliorganversagen}

\section{Definition}

Als Multiorganversagen (MOV) bezeichnet man das gleichzeitig oder in rascher zeitlicher Abfolge auftretende Versagen von zwei oder mehr vitalen Organsystemen (2). In der Regel gehen dem manifesten Organversagen Zeichen einer initialen Organinsuffizienz voraus. Die insuffiziente Leistung eines Organs wird als Organdysfunktion, das Zusammentreffen dieser Situation für mehrere Organe als Multiorgan-Dysfunktionssyndrom (MODS) bezeichnet. In der klinischen Anwendung ist eine Unterscheidung entsprechend diesen Definitionen häufig nicht klar zu treffen, da bei einem Patienten durchaus ein oder zwei Organe Zeichen einer Dysfunktion zeigen können, während ein weiteres Organ bereits versagt. Dieser Problematik trägt die zunehmende Verwendung des Begriffes des MODS auch für Patienten, die neben einer Organdysfunktion zusätzlich ein Versagen von ein oder mehreren Organen zeigen, Rechnung.

Klinisch die häufigste Ursache für die Entwicklung eines MODS bei kritisch Kranken ist die bakterielle Sepsis. Die Organfunktionsstörungen, die bereits in der Initialphase der Sepsis auftreten, können mit Progression des Krankheitsverlaufs bis zum multiplen Organversagen fortschreiten. Ein Multiorganversagen ist der klinische Endpunkt des fortschreitenden septischen Prozesses.

Die hauptsächlich betroffenen Organsysteme (Tab. 4) und damit die Hauptkomponenten von Multiorganinsuffizienz und Multiorganversagen sind:

akute respiratorische Insuffizienz und akutes Lungenversagen (ARDS)

akute kardiozirkulatorische Insuffizienz und Herz-KreislaufSchock akute renale Insuffizienz und akutes Nierenversagen akute Leberinsuffizienz und akutes Leberversagen akute gastrointestinale Läsionen und Stressblutungen 
Tab. 4 Diagnostische Kriterien des Multiorganversagens

\begin{tabular}{|c|c|}
\hline $\begin{array}{l}\text { Akutes Lur } \\
\text { versagen }\end{array}$ & $\begin{array}{l}\text { Hypoxämie ( } \mathrm{paO}_{2} \text { unter Altersnorm bei Atmung von Raumluft), } \\
\text { pathologischer radiologischer Befund im Thoraxbild, } \\
\text { Respiratortherapie erforderlich }\end{array}$ \\
\hline $\begin{array}{l}\text { Herzversagen, } \\
\text { Kreislaufschock }\end{array}$ & $\begin{array}{l}\text { Arterielle Hypotension trotz Volumensubstitution, } \\
\text { Katecholamine erforderlich }\end{array}$ \\
\hline $\begin{array}{l}\text { Akute } \\
\text { versa }\end{array}$ & $\begin{array}{l}\text { Anstieg Serumkreatinin }>3 \mathrm{mg} / \mathrm{dl} \text {, } \\
\text { Kreatinin-Clearance }<15 \mathrm{ml} / \mathrm{min} \times 1,73 \mathrm{~m}^{2} \text { trotz Normalisie- } \\
\text { rung von Blutdruck und Flüssigkeitshaushalt, } \\
\text { Nierenersatzverfahren erforderlich }\end{array}$ \\
\hline $\begin{array}{l}\text { Akutes Leber- } \\
\text { versagen }\end{array}$ & $\begin{array}{l}\text { Anstieg Serumbilirubin }>2 \mathrm{mg} / \mathrm{ml} \text {, } \\
\text { Erhöhung Transaminasen }>2 \text {-Faches der Norm }\end{array}$ \\
\hline $\begin{array}{l}\text { Gastrointestinale } \\
\text { Stressblutung }\end{array}$ & $\begin{array}{l}\text { Endoskopisch Erosionen oder Ulzera, Bluttransfusion erforder- } \\
\text { lich }\end{array}$ \\
\hline $\begin{array}{l}\text { Disseminierte } \\
\text { intravasale } \\
\text { Gerinnung (DIC) }\end{array}$ & $\begin{array}{l}\text { Abfall Thrombozytenzahl (Thrombopenie oder rascher Abfall } \\
\text { um } 150000 / \mathrm{mm}^{3} \text { ), } \\
\text { Abfall der Fibrinogenkonzentration (Hypofibrinogenämie oder } \\
\text { rascher Abfall um } 150 \text { mg/dl), pathologische plasmatische } \\
\text { Gerinnungstests, mindestens zwei (Quick, PTा, TT, Faktor II, V, X) }\end{array}$ \\
\hline
\end{tabular}

- akute akalkulöse Cholezystitis und Gallenperforation akute Enterokolitis, akute Pankreatitis

akute Blutgerinnungsstörungen und disseminierte intravasale Gerinnung

akute Störung des Bewusstseins und metabolisches Koma akute Störungen des autonomen und peripheren Nervensystems sowie der Skelettmuskulatur

\section{Diagnose}

Im Gegensatz zu Sepsis und SIRS stehen einheitliche diagnostische Kriterien für das Vorliegen eines MODS oder MOV von Seiten der nationalen und internationalen Gesellschaften noch aus. Auch die amerikanische Konsensus-Konferenz hat festgestellt, dass solche vereinheitlichten diagnostischen Kriterien fehlen.

Die Schwere eines MODS wird durch Angabe der Zahl der betroffenen Organsysteme erfasst. Innerhalb einzelner Organsysteme ist die Unterscheidung des Schweregrades der Funktionsstörung anhand von MODS- und MOV-Scores möglich $(14,21)$. Der hierfür am häufigsten angewandte Score ist der „Multiple Organ Failure“ (MOF) Score nach Goris. Eine Graduierung des Sepsis-induzierten MODS ist anhand des SOFA-Scores möglich (siehe dazu (14)). Bei Patienten mit MOV in Folge einer Sepsis wird ein septischer Schock in etwa 75\% der Fälle beobachtet. Dabei ist der septische Schock eher Teil des MOV als ein essentieller Schritt in dessen Pathogenese.

\section{MODS - nur die Summe einzelner Organdysfunktionen oder} Folge einer gestörten Organinteraktion?

Die rein summarische Auflistung der dysfunktionierenden Organe lässt die Organinteraktionen als wesentlichen Aspekt für die Entwicklung und Aufrechterhaltung der Homöostase und andererseits wesentlichen pathogenetischen Aspekt eines MODS außer Acht. So ist bei kritisch Kranken eine eingeschränkte Herzfrequenzvariabilität Ausdruck einer Dysbalance zwischen Sympathikus- und Parasympathikus-Aktivität und geht mit einer 13-fach erhöhten Letalität einher (20). Der Grad der Einschränkung der Herzfrequenzvariabilität als Ausdruck der autonomen Dysfunktion scheint direkt zum Schweregrad der Erkrankung korreliert. In einer Pilotstudie zeigten Patienten mit einem MODS septischer Genese eine deutliche Einschränkung der Herzfrequenzvariabilität gegenüber nicht-septischen Intensivpatienten ohne MODS (10). Diese Ergebnisse unterstreichen die Bedeu- tung eines über die Einschränkung der isolierten Organfunktion hinausgehenden Verlustes der nerval-humoral vermittelten Organinteraktion für die Ausprägung eines MODS. Ob und inwieweit eine gestörte nerval-humoral vermittelte Organinteraktion - die auch interpretiert wird als eine Entkopplung biologischer Oszillatoren - als eigenständiger Risikofaktor für die Entwicklung eines und Prognose eines MODS gewertet werden muss (7), werden künftige Untersuchungen belegen müssen.

kurzgefasst: Dem Mulitorganversagen geht eine Multiorgan-Dysfunktion (MODS) voraus, die Übergänge können fließend sein. Die Schwere eines MODS richtet sich nach der Anzahl der betroffenen Organsysteme. Der Grad der Einschränkung der Herzfrequenzvariabilität als Ausdruck der autonomen Dysfunktion scheint direkt zum Schweregrad der Erkrankung korreliert.

Autorenerklärung: Die Autorin leitet derzeit ein wissenschaftliches Projekt, das von der Firma Biotest unterstützt wird, und war in der Vergangenheit mehrfach an Industrie-geförderten Forschungsvorhaben beteiligt bzw. leitete solche Projekte.

\section{Literatur}

Al Nawas B. Krammer I, Shah PM. Procalcitonin in diagnosis of severe infections. Eur J Med Res 1996; 1: 331-333

2 Baue AE. Multiple, progressive, or sequential systems failure. A syndrome of the 1970s. Arch Surg 1975; 110: 779-781

Bone RC. Sir Isaak Newton, sepsis, SIRS, and CARS. Crit Care Med 1996; 24: 11251128

4 De Werra I, Jaccard C, Betz Corradin S et al. Cytokines, nitrite/nitrate, soluble tumor necrosis factor receptors, and procalcitonin concentrations: Comparison in patients with septic shock, cardiogenic shock, and bacterial pneumonia. Crit Care Med 1997; 25: 607-613

5 Fraunberger P, Walli AK, Seidel D. Stellenwert von Zytokinen in der Sepsis-Diagnostik. Infusionsther Transfusionsmed 1996; 23 (Suppl 4); 109-116

6 Giamarellos-Bourboulis E, Mega A, Grecka P et al. Procalcitonin: a marker to clearly differentiate systemic inflammatory response syndrome and sepsis in the clearly differentiate systemic inflammatory response syndrom Godin P, Buchmann TC. Uncoupling of biological oscillators. A complementary hypothesis concerning the pathogenesis of multiple organ dysfunction syndro-

8 Harbarth S, Holeckova K, Froidevaux Cet al. Diagnostic value of procalcitonin, interleukin-6, and interleukin-8 in critically ill patients admitted with suspected sepsis. Am J Respir Crit Care Med 2001; 164: 396-402

Members of the American College of Chest Physician/Society of Critical Care Medicine Consensus Conference Committee. Definition for sepsis and organ failure and guidelines for the use of innovative therapies in sepsis. Crit Care Med 1992; 20: 864-874

10 Müller-Werdan U, Prondzinsky R, Witthaut R, Stache N, Heinroth K, Kuhn C, Schmidt H, Busch I, Werdan K. Das Herz bei Sepsis und MODS. Wien Klin Wochenschr 1997; 109 (Suppl 1); 3-24

11 Panacek EA, Marshall J, Fischkoff S et al. Neutralization of TNF by a monoclonal antibody improves survival and reduces organ dysfunction in human sepsis: Results of the MONARCS trial. Chest 2000; 118: 88

12 Pilz G, Fraunberger P, Appel R, Kreuzer E, Werdan K, Walli A, Seidel D. Bedeutung erhöhter TNF-Rezeptorspiegel bei herzchirurgischen Risikopatienten. Infusionsther Transfusionsmed 1996; 23 (Suppl 4); 29-34

13 Pilz G, Fraunberger P, Appel R, Kreuzer E, Werdan K, Walli A, Seidel D. Early prediction of outcome in score-identified, postcardiac patients at high risk for sepsis, using soluble tumor necrosis factor receptor-p55 concentrations. Crit Care Med 1996; 24: 596-600

14 Pilz G, Werdan K. Scores für Multiorgandysfunktion und Multiorganversagen. Internist 1998; 39: 502-508

15 Reinhart K, Meisner M, Hartog C. Diagnosis of sepsis: Novel and conventional parameters. Advances in Sepsis 2001; 1: 42-51

16 Reinke P, Döcke W-D, Kox W et al Neue Entwicklungen in der Immundiagnostik Reinke P, Döcke W-D, Kox W et al. Neue Entwicklungen in der Immundiagnostik

intensivmedizinischer Patienten. Dtsch Med Wochenschr 1999; 124: 1527-1529 incent J-L, Moreno R, Takala J et al. The SOFA (Sepsis-related Organ Failure As22: 707-710

Vincent J-L. Dear SIRS, I'm sorry to say that I don't like you. Crit Care Med 1997;

Werdan K, Müller-Werdan U. Sektion VI. Schock. 5. vollk. überarb. Auflage (Hrsg). Springer-Verlag Heidelberg New York, In: Erdmann E (Hrsg) Klinische Karchologie. 2000: 703-796

Winchell RJ, Hoyt DB. Spectral analysis of heart rate variability in the ICU. J Surg Res 1996; 63: 11-16

21 Witthaut R, Werdan K, Schuster H-P. Multiorgandysfunktions-Syndrom und Multiorganversagen - Diagnose, Prognose und Therapiekonzepte. Internist 1998; 39: 493-501

22 Witthaut R, Werdan K. Ergebnisforschung am Beispiel der Sepsis. Internist 1996 37: 1249-1259 